\title{
Mechanism of Mitral Regurgitation in the Acute Phase of Inferior Wall Myocardial Infarction \\ - Reduced Closing Force as a Consequence of Left Ventricular Systolic Dysfunction in the Presence of Tethering as a Determinant of Mitral Regurgitation -
}

Sung-Jin Hong, MD; Sung-Ai Kim, MD; Jin Hur, MD; Sang-Jae Rhee, MD; Chi Young Shim, MD; Jung-Sun Kim, MD; Young-Guk Ko, MD; Eui-Young Choi, MD; Donghoon Choi, MD, PhD; Yangsoo Jang, MD, PhD; Namsik Chung, MD, PhD; Jong-Won Ha, MD, PhD

\begin{abstract}
Background: To delineate the mechanism of mitral regurgitation (MR) in the acute phase of inferior wall myocardial infarction (MI).
\end{abstract}

\begin{abstract}
Methods and Results: In 97 patients (age 59 \pm 12 years) with acute inferior wall MI, the severity of MR, left ventricular (LV) geometric changes and function within 1.7 \pm 1.3 days after MI by echocardiography was retrospectively evaluated. Infarct size was measured within $3.9 \pm 1.7$ days by cardiac magnetic resonance. Mild MR was present in $16(16.5 \%)$ and moderate MR in $12(12.4 \%)$ of 97 patients. There were no significant differences in mitral annular area, sphericity and distances between papillary muscle tips and the contralateral mitral annulus. However, patients with moderate MR had significantly larger LV end-systolic volume, tenting area and infarct size. There was a graded relationship between the severity of MR and LV ejection fraction. In a multivariable regression analysis, LV ejection fraction and tenting area were found to be independent predictors of the severity of $M R\left(r^{2}=0.180, P=0.001\right)$.

Conclusions: MR was associated with LV systolic dysfunction, increase in end-systolic LV volume and tethering of mitral leaflet, suggesting reduced closing force as a consequence of LV systolic dysfunction in the presence of leaflet tethering would play a more pivotal role in the development of MR. (Circ J 2011; 75: 619-625)
\end{abstract}

Key Words: Mitral valve; Myocardial infarction; Regurgitation

$\mathbf{I}$ schemic mitral regurgitation (MR) is MR due to complications of coronary artery disease and not with intrinsic valve disease such as rheumatic or degenerative mitral valvular disease. ${ }^{1}$ It is common and clinically important because it increases mortality even when mild, with a graded relationship between severity and reduced survival. ${ }^{2-4}$ Ventricular remodeling with papillary muscle (PM) displacement has been known to be an important mechanism of ischemic MR, especially in patients with inferior wall myocardial infarction (MI). Therefore, MR occurs at a higher incidence in patients with inferior MI compared with those with anterior MI, despite its less severe left ventricular (LV) remodeling, because of the greater displacement of posterior PM caused by localized inferior basal LV remodeling. ${ }^{5-7}$ How- ever, most of the previous studies excluded the patients with MI in the acute phase. Thus, the mechanism of MR during acute phase of inferior MI is unclear. We hypothesized the mechanism of MR during acute phase could be different from that of MR during chronic phase and there could be more contributing factors than local remodeling in patients with inferior MI because of the influence of different chronicity of MI and the diversity of mechanism of MR. Thus, we compared geometric changes and LV function by echocardiography between patients with or without MR.

Cardiac magnetic resonance (CMR) allows a complete and accurate assessment of LV status in patients after acute MI and late gadolinium-enhanced CMR visualizes and quantifies infarct size. ${ }^{89}$ However, few studies investigated the

Received August 24, 2010; revised manuscript received October 1, 2010; accepted November 4, 2010; released online January 8, 2011 Time for primary review: 14 days

Cardiology Division (S.-J.H., S.-A.K., S.-J.R., C.Y.S., J.-S.K., Y.-G.K., E.-Y.C., D.C., Y.J., N.C., J.-W.H.), Department of Radiology

(J.H.), Yonsei University College of Medicine, Seoul, South Korea

Funding: Korea Health 21 R\&D Project, Ministry of Health and Welfare, Republic of Korea (0412-CR02-0704-0001).

Mailing address: Jong-Won Ha, MD, PhD, FESC, Cardiology Division, Severance Cardiovascular Hospital, Yonsei University College of Medicine, Seodaemun-Gu Shinchon-Dong 134, 120-752, Seoul, South Korea. E-mail: jwha@yuhs.ac

ISSN-1346-9843 doi:10.1253/circj.CJ-10-0859

All rights are reserved to the Japanese Circulation Society. For permissions, please e-mail: cj@j-circ.or.jp 


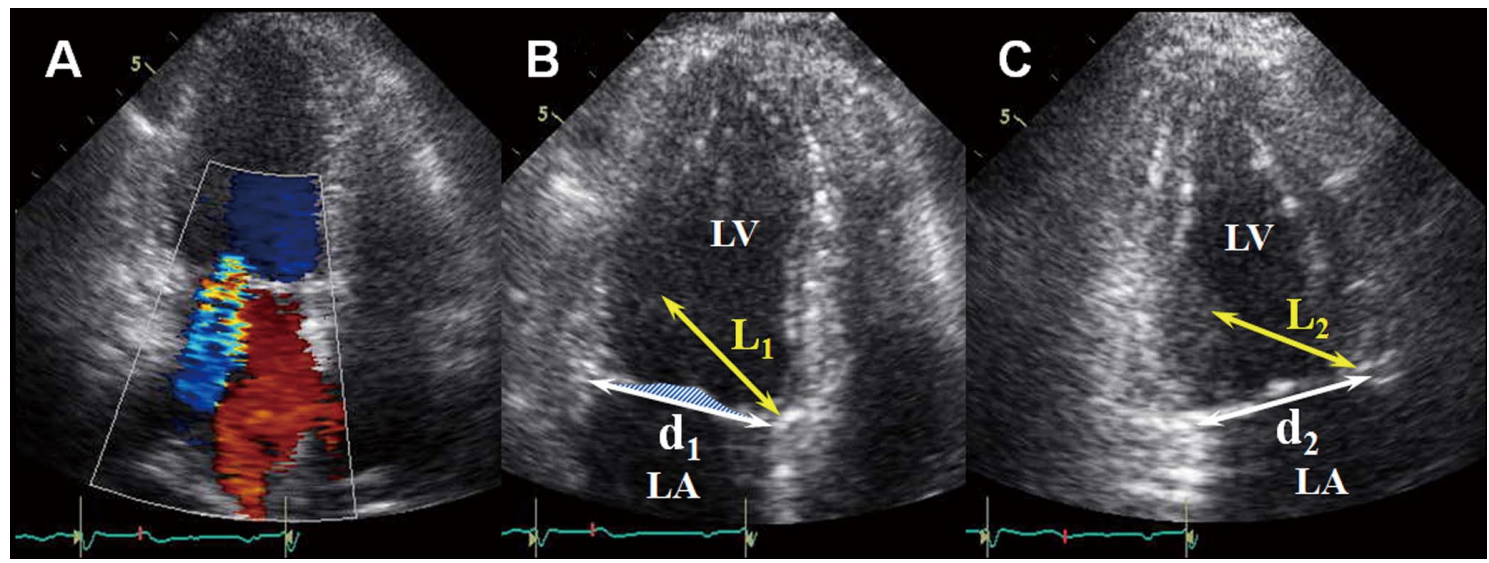

Figure 1. Methods to measure (1) the mitral annular area from 2 diameters in apical views ( $d_{1}$ and $\left.d_{2}\right)$, (2) the mitral leaflet-tenting area between the mitral leaflets and a line connecting mitral annular hinge points in the midsystolic apical 4-chamber view (shaded area shown in B), and (3) leaflet-tethering lengths between anterior or posterior papillary muscle (PM) tips and contralateral anterior mitral annulus ( $\mathrm{L}_{1}$ and $\left.\mathrm{L}_{2}\right)$. LV, left ventricle; $L A$, left atrium

relationship between infarct size by $\mathrm{CMR}$ and the severity of MR. We thought that there would be a positive association between the severity of MR and infarct size if LV remodeling is an important mechanism of MR. We compared infarct size by CMR between patients with or without MR to investigate the association of infarct size with MR.

\section{Methods}

\section{Patients}

Between January 2000 and May 2010, 218 patients with acute inferior wall MI who had undergone a CMR were enrolled retrospectively. Acute MI was diagnosed by the presence of at least 2 of the following criteria: electrocardiographic changes, significant rises in myocardial bound creatine kinase fraction, and typical chest pain. Inferior wall MI was diagnosed by electrocardiography, echocardiography and coronary angiography. In patients with non-ST elevation MI, echocardiography and coronary angiographic findings were used for determination of the diagnosis of inferior wall MI.

In 93 of 218 consecutive patients, echocardiography was not performed within 5 days or CMR within 7 days after acute MI. Twenty-eight patients were also excluded because they had MR caused by intrinsic mitral valvular lesions (including rheumatic changes, infective endocarditis, and mitral valve prolapse), other associated cardiac diseases, PM rupture due to MI, history of previous MI, abnormal wall motions in the anterior wall by echocardiography or hyper-enhancement areas (infarct areas) in the anterior wall by CMR. Thus, echocardiographic measurements were available in 97 patients. CMR imagings were available in $89(92 \%)$ of 97 patients. Of 8 patients, 5 patients had other pulse sequences taken with a different scanner and thus images were not suitable to analyze with our software program (ViewForum, version 4.1, Philips Medical Systems, Netherlands), 2 patients did not meet the inversion time when taking a CMR and another patient's infarct area analysis was not possible because of respiratory motion.

\section{Echocardiographic Measurements}

LV end-diastolic and end-systolic cavity was traced in the apical 4-chamber and 2-chamber views, and LV volume was obtained by using the modified biplane Simpson method. ${ }^{10}$ LV ejection fraction was calculated from the LV end-diastolic and end-systolic volumes. Regional wall motion was assessed by assigning a segmental score to each of the $17 \mathrm{LV}$ segments. ${ }^{11}$ All segment scores were added and divided by the number of segments analyzed to obtain the regional wall motion score index (RWSI). ${ }^{10} \mathrm{LV}$ sphericity was assessed by using the LV short-axis/long-axis dimension ratio in the endsystolic apical 4-chamber view. ${ }^{12}$ Left atrial (LA) volume was calculated using the prolate ellipsoid model. ${ }^{13}$ From mitral Doppler tracings with the sample volume at the mitral leaflet tips, the following variables were measured: peak velocity of early rapid filling wave (E), peak flow velocity at atrial contraction (A), E/A ratio, and deceleration time of early filling. A restrictive LV filling pattern was defined as an E/A ratio $>2$, with a deceleration time of $<150 \mathrm{~ms} .{ }^{14}$ Early and late diastolic tissue Doppler velocities (E' and A') were measured at the medial mitral annulus using a tissue Doppler image.

Midsystolic mitral annular dimension was measured in the apical 4- and 2-chamber views, and its area was calculated by using an ellipsoid assumption (annular area $=\mathrm{d}_{1} \times \mathrm{d}_{2} \times \pi / 4$ ) (Figure 1). ${ }^{15,16}$ The mitral leaflet-tenting area between the leaflets and the line connecting the annular hinge points in the apical 4-chamber view was traced at midsystole to estimate the apical displacement of the mitral leaflets (Figure 1B). ${ }^{17}$ The leaflet-tethering distance between the PM tips and the contralateral anterior mitral annulus was also measured in the apical 4- and 2-chamber views (Figure 1, $\mathrm{L}_{1}$ and $\mathrm{L}_{2}$ ) to estimate PM displacement. ${ }^{18-20}$ The severity of MR was determined by the ratio of color Doppler jet area to LA area at midsystole. MR grade was estimated as mild, moderate, or severe on the basis of ratios of greater than $10 \%$ to $20 \%$, greater than $20 \%$ to $40 \%$, and greater than $40 \%$, respectively. ${ }^{21,22}$

\section{CMR Easurements}

CMR was performed within $3.9 \pm 1.7$ days (range 1-7) after 


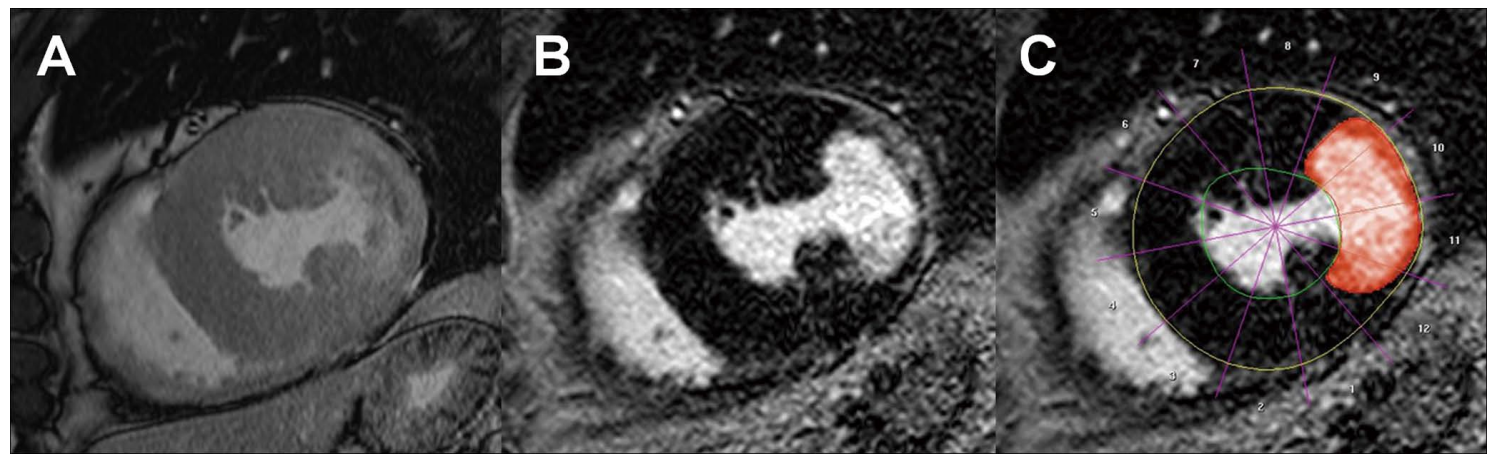

Figure 2. Cardiac magnetic resonance (CMR) assessment of left ventricle (LV) mass and infarct area. (A) Steady-state free precession image to discriminate between blood and endocardium. (B) Delayed enhancement image shows infarct area. (C) Epicardial and endocardial contours of the entire LV were manually traced for determining the LV mass (yellow and green line). Each slice was divided into 12 circumferential segments (purple line) to determine the infarct transmurality. The region of hyper-enhancement area (infract area) was semi-automatically defined by the software program using automatic thresholding technique (red-shaded area).

\begin{tabular}{|c|c|c|c|c|}
\hline MR & $\begin{array}{l}\text { None } \\
(n=69)\end{array}$ & $\begin{array}{c}\text { Mild } \\
(n=16)\end{array}$ & $\begin{array}{c}\text { Moderate } \\
(n=12)\end{array}$ & $P$ value \\
\hline Age (years) & $58.9 \pm 12.1$ & $58.9 \pm 9.9$ & $62.4 \pm 11.3$ & 0.460 \\
\hline Male & $55(79.7 \%)$ & $13(81.3 \%)$ & $7(58.3 \%)$ & 0.243 \\
\hline $\mathrm{BSA}\left(\mathrm{m}^{2}\right)$ & $1.79 \pm 0.18$ & $1.77 \pm 0.15$ & $1.65 \pm 0.13$ & 0.031 \\
\hline Body mass index $\left(\mathrm{kg} / \mathrm{m}^{2}\right)$ & $25.0 \pm 3.3$ & $23.9 \pm 2.5$ & $23.4 \pm 2.6$ & 0.177 \\
\hline STEMI & $31(44.9 \%)$ & 9 (56.3\%) & $4(33.3 \%)$ & 0.479 \\
\hline Hypertension & $27(39.1 \%)$ & $8(50.0 \%)$ & $4(33.3 \%)$ & 0.635 \\
\hline Diabetes mellitus & $17(24.6 \%)$ & $2(12.5 \%)$ & $3(25.0 \%)$ & 0.567 \\
\hline Renal disease & $5(7.2 \%)$ & $0(0 \%)$ & $2(16.7 \%)$ & 0.241 \\
\hline Smoking & $28(40.6 \%)$ & 9 (56.3\%) & $4(33.3 \%)$ & 0.416 \\
\hline Dyslipidemia & $28(40.6 \%)$ & 7 (43.8\%) & $3(25.0 \%)$ & 0.546 \\
\hline \multicolumn{5}{|c|}{ Severity of coronary artery disease } \\
\hline 1-vessel & $28(40.6 \%)$ & $6(37.5 \%)$ & $3(25.0 \%)$ & \\
\hline 2-vessel & $21(30.4 \%)$ & $5(31.3 \%)$ & $5(41.7 \%)$ & \\
\hline 3-vessel & $20(29.0 \%)$ & $5(31.3 \%)$ & $4(33.3 \%)$ & \\
\hline \multicolumn{5}{|l|}{ Culprit lesion } \\
\hline Right coronary artery & $53(76.8 \%)$ & $10(62.5 \%)$ & $8(66.7 \%)$ & 0.437 \\
\hline Left circumflex artery & $16(23.2 \%)$ & $6(37.5 \%)$ & $4(33.3 \%)$ & \\
\hline \multicolumn{5}{|l|}{ Lipid profile } \\
\hline Total cholesterol & $179.3 \pm 42.7$ & $180.9 \pm 33.5$ & $165.3 \pm 48.5$ & 0.540 \\
\hline LDL cholesterol & $116.7 \pm 39.0$ & $109.3 \pm 26.3$ & $111.0 \pm 43.7$ & 0.764 \\
\hline HDL cholesterol & $37.8 \pm 8.0$ & $41.3 \pm 11.2$ & $38.5 \pm 6.0$ & 0.348 \\
\hline \multicolumn{5}{|l|}{ Cardiac enzyme } \\
\hline Initial CK-MB & $25.7 \pm 66.4$ & $25.7 \pm 51.1$ & $47.64 \pm 76.7$ & 0.559 \\
\hline Peak CK-MB & $193.6 \pm 156.2$ & $200.0 \pm 241.3$ & $232.6 \pm 288.6$ & 0.810 \\
\hline
\end{tabular}

MR, mitral regurgitation; BSA, body surface area; STEMI, ST elevation myocardial infarction; LDL, low-density lipoprotein; HDL, high-density lipoprotein; CK-MB, myocardial bound creatine kinase.

acute MI using a 1.5 Tesla (T) imaging unit (Gyroscan Intera, Philips Medical Systems, Netherlands) equipped with a dedicated cardiac software package and a dedicated cardiac phased-array surface coil. Delayed enhancement images was performed using a segmented inversion recovery radiofrequency spoiled gradient echo (T1-TFE) sequence (typical $\mathrm{TR} / \mathrm{TE}=5.3 / 1.6 \mathrm{~ms}$, flip angle $=15^{\circ}$, slice thickness $=10 \mathrm{~mm}$, field of view $=360 \mathrm{~mm}$, matrix size $=512 \times 512$, number of signal average =2) $10 \mathrm{~min}$ after the intravenous injection of gadolinium-diethylene-triamine-pentaacetic acid at a dose of $0.2 \mathrm{mmol} / \mathrm{kg}$ body weight. The inversion time was determined by a dedicated TI-determining sequence (LookLocker) and ranged from 220 to $300 \mathrm{~ms}$. Contiguous end-diastolic short-axis slices of the LV were acquired from base to apex without gaps (8-10 slices in number) to cover the whole LV (Figure 2B). For quantitative analysis, we used the scan- 


\begin{tabular}{|c|c|c|c|c|}
\hline MR & $\begin{array}{l}\text { None } \\
(n=69)\end{array}$ & $\begin{array}{c}\text { Mild } \\
(n=16)\end{array}$ & $\begin{array}{c}\text { Moderate } \\
(n=12)\end{array}$ & $P$ value \\
\hline \multicolumn{5}{|l|}{ Mitral deformation } \\
\hline Tenting area/BSA $\left(\mathrm{cm}^{2} / \mathrm{m}^{2}\right)$ & $0.61 \pm 0.21$ & $0.79 \pm 0.23$ & $0.80 \pm 0.21$ & 0.002 \\
\hline Annular area/BSA $\left(\mathrm{cm}^{2} / \mathrm{m}^{2}\right)$ & $4.19 \pm 1.31$ & $4.23 \pm 1.27$ & $4.02 \pm 1.34$ & 0.907 \\
\hline \multicolumn{5}{|c|}{ LV global and local systolic function } \\
\hline LV ejection fraction (\%) & $52.1 \pm 10.6$ & $48.0 \pm 9.6$ & $41.7 \pm 11.6$ & 0.003 \\
\hline Total RWSI & $1.41 \pm 0.29$ & $1.54 \pm 0.37$ & $1.66 \pm 0.23$ & 0.022 \\
\hline Inferior RWSI & $1.87 \pm 0.65$ & $2.08 \pm 0.64$ & $2.15 \pm 0.57$ & 0.239 \\
\hline Posterior RWSI & $1.45 \pm 0.53$ & $1.64 \pm 0.78$ & $1.88 \pm 0.64$ & 0.053 \\
\hline \multicolumn{5}{|l|}{ LV diastolic function } \\
\hline $\mathrm{E}(\mathrm{cm} / \mathrm{s})$ & $73.3 \pm 18.7$ & $76.4 \pm 18.1$ & $68.1 \pm 19.1$ & 0.507 \\
\hline $\mathrm{A}(\mathrm{cm} / \mathrm{s})$ & $68.5 \pm 20.6$ & $66.2 \pm 23.1$ & $77.3 \pm 32.0$ & 0.392 \\
\hline $\mathrm{E} / \mathrm{A}$ & $1.20 \pm 0.64$ & $1.30 \pm 0.53$ & $0.95 \pm 0.28$ & 0.282 \\
\hline Deceleration time (ms) & $178.1 \pm 34.6$ & $172.8 \pm 37.9$ & $169.3 \pm 31.3$ & 0.658 \\
\hline Restrictive LV filling & $3(4.3 \%)$ & $1(6.3 \%)$ & $1(8.3 \%)$ & 0.827 \\
\hline$E^{\prime}(\mathrm{cm} / \mathrm{s})$ & $6.0 \pm 1.7$ & $5.3 \pm 1.5$ & $5.2 \pm 1.9$ & 0.213 \\
\hline$A^{\prime}(\mathrm{cm} / \mathrm{s})$ & $7.8 \pm 1.6$ & $7.1 \pm 2.3$ & $6.8 \pm 2.2$ & 0.234 \\
\hline $\mathrm{E} / \mathrm{E}^{\prime}(\mathrm{cm} / \mathrm{s})$ & $13.1 \pm 5.3$ & $14.7 \pm 5.5$ & $15.8 \pm 7.9$ & 0.341 \\
\hline \multicolumn{5}{|l|}{ LV global remodeling } \\
\hline Sphericity & $0.47 \pm 0.09$ & $0.46 \pm 0.08$ & $0.44 \pm 0.11$ & 0.698 \\
\hline LVEDVI $\left(\mathrm{ml} / \mathrm{m}^{2}\right)$ & $52.9 \pm 16.1$ & $60.6 \pm 16.2$ & $66.4 \pm 35.8$ & 0.079 \\
\hline LVESVI $\left(\mathrm{ml} / \mathrm{m}^{2}\right)$ & $25.3 \pm 8.8$ & $29.7 \pm 10.4$ & $34.7 \pm 16.8$ & 0.018 \\
\hline \multicolumn{5}{|l|}{ LV local remodeling } \\
\hline Annular to APM/BSA $\left(\mathrm{mm} / \mathrm{m}^{2}\right)$ & $26.2 \pm 4.1$ & $28.0 \pm 2.9$ & $27.9 \pm 5.3$ & 0.182 \\
\hline Annular to PPM/BSA $\left(\mathrm{mm} / \mathrm{m}^{2}\right)$ & $25.0 \pm 3.5$ & $25.9 \pm 3.3$ & $26.2 \pm 4.5$ & 0.468 \\
\hline \multicolumn{5}{|l|}{ LA remodeling } \\
\hline LAVI $\left(\mathrm{ml} / \mathrm{m}^{2}\right)$ & $26.1 \pm 6.4$ & $30.3 \pm 5.4$ & $30.5 \pm 7.4$ & 0.014 \\
\hline
\end{tabular}

LV, left ventricle; RWSI, regional wall motion score index; LVEDVI, left ventricular end-diastolic volume index; LVESVI, left ventricular end-systolic volume index; APM, anterior papillary muscle; PPM, posterior papillary muscle; LAVI, left atrial volume index. Other abbreviations see in Table 1.

ner's workstation using commercially available software (ViewForum, version 4.1, Philips Medical Systems). Epicardial and endocardial contours of the entire LV were manually traced for determining the LV mass (Figure 2C, yellow and green line). Each slice was divided into 12 circumferential segments on up to 6 short axis views (Figure 2C, purple line). The region of hyper-enhancement area (infract area) was semi-automatically defined by the software program using automatic thresholding technique (Figure 2C, redshaded area). Manual adjustments of the infarct region of interest were made where the computer algorithm failed to correctly delineate the infarct area. The same density $\left(1.05 \mathrm{~g} / \mathrm{cm}^{3}\right)$ was assumed for both hyperenhanced and nonhyperenhanced myocardium. ${ }^{23} \mathrm{LV}$ mass and infarct mass were indexed to body surface area. Infarct size was defined as the total amount of hyper-enhancement area in all shortaxis slices and expressed as a percentage of LV mass. The extent of hyperenhanced area within each segment (referred to as the infarct transmurality) was defined as percent of the hyperenhanced myocardium to total area of the involved segment of the myocardium. Infarct transmurality was defined as percent of the hyper-enhanced myocardium to total area of the involved myocardium and transmurality index was graded as $1,2,3$, or 4 based on its occupation of $1-25 \%, 26-50 \%$, $51-75 \%$, or $76-100 \%$ of the myocardium, respectively. The mean transmurality index for each patient was calculated as the average of all segments with of a grade $>1$.

\section{Statistical Analysis}

All continuous variables were presented as mean \pm standard deviation and compared by analysis of variance. Discrete variables were compared using $\chi^{2}$ analysis or the Fisher exact test, as appropriate. Independent correlates of the severity of MR were identified by forward stepwise multivariable regression (forward method, with $\mathrm{P}<0.05$ for entrance into the model and $\mathrm{P}>0.10$ for removal from the model). The variables entered in the multivariable model were LV ejection fraction, tenting area, LV end-systolic volume index and infarct size, which were significant variables in the univariate analysis. A value of $\mathrm{P}$ less than 0.05 was considered significant.

\section{Results}

Echocardiography was performed within $1.7 \pm 1.4$ days (range 0-5) after acute MI. MR was present in 28 (29\%) of 97 patients; mild in 16 patients, moderate in 12 and there were no patients with severe MR. Direction of MR jet was central in $10(63 \%)$ patients with mild MR and in $8(67 \%)$ patients with moderate MR. The other patients' direction of MR was eccentric. Clinical characteristics are summarized in Table 1. There were no differences in age, sex and other frequencies of underlying diseases among the 3 groups. There were no differences in the modality of intervention, severity of coronary artery disease and cardiac enzymes. Seventy-one (73.2\%) patients had the culprit lesion in the right coronary artery and $26(26.8 \%)$ patients had the culprit lesion in the left circum- 


\begin{tabular}{|c|c|c|c|c|}
\hline MR & $\begin{array}{l}\text { None } \\
(n=63)\end{array}$ & $\begin{array}{c}\text { Mild } \\
(n=15)\end{array}$ & $\begin{array}{c}\text { Moderate } \\
(n=11)\end{array}$ & $P$ value \\
\hline Total LV mass index $\left(\mathrm{g} / \mathrm{m}^{2}\right)$ & $65.5 \pm 14.6$ & $68.9 \pm 17.9$ & $71.9 \pm 21.1$ & 0.409 \\
\hline Infarct mass index $\left(\mathrm{g} / \mathrm{m}^{2}\right)$ & $11.8 \pm 7.7$ & $16.1 \pm 8.5$ & $18.6 \pm 9.8$ & 0.018 \\
\hline Infarct size (\%) & $17.4 \pm 9.6$ & $22.2 \pm 8.4$ & $25.9 \pm 12.1$ & 0.016 \\
\hline Infarct transmurality & $2.95 \pm 0.54$ & $3.06 \pm 0.40$ & $3.35 \pm 0.49$ & 0.064 \\
\hline
\end{tabular}

Abbreviations see in Tables 1,2.

flex artery. Patients whose culprit lesion in the left circumflex artery had an increased frequency of more severe MR than those with the culprit lesion in the right coronary artery, but this difference did not reach statistical significance $(\mathrm{P}=0.437)$. Four patients without MR had right ventricular infarct but any patients with MR did not have right ventricular involvement.

\section{Mitral Deformation}

There were no significant differences in annular area within the 3 groups $(\mathrm{P}=0.907)$. However, patients with mild or moderate MR had larger tenting area than those without MR (0.79 or 0.80 vs. $0.61, \mathrm{P}=0.004$ and 0.007 ) (Table 2).

\section{Systolic and Diastolic Function}

Regarding LV systolic function, there was a graded relationship between the severity of MR and LV ejection fraction $(\mathrm{P}=0.003)$. Patients with moderate MR had more decreased LV systolic function than those without MR (41.7\% vs. $52.1 \%, \mathrm{P}=0.001)$. Patients with moderate MR had significantly more increased total RWSI than those without MR (1.66 vs. $1.41, \mathrm{P}=0.005)$ and patients with moderate $\mathrm{MR}$ had decreased wall motion at inferior/posterior wall than those without MR but this did not reach a statistical significance in the inferior wall (inferior RWSI 2.15 vs. $1.87, \mathrm{P}=0.239$ and posterior RWSI 1.88 vs. $1.45, \mathrm{P}=0.053$ ) (Table 2).

With regard to LV diastolic function, there were no significant differences in $\mathrm{E}, \mathrm{A}, \mathrm{E} / \mathrm{A}$, deceleration time of $\mathrm{E}$ velocity, E', A' and E/E' between the 3 groups. There were also no significant differences in the frequency of restrictive LV filling $(\mathrm{P}=0.827)$ (Table 2).

\section{Global and Local Remodeling}

With regard to LV global remodeling, there were no significant differences in sphericity of LV. However, there was a graded relationship between the severity of MR and LV dimension. Patients with moderate MR had larger end-systolic volume than those with mild MR and without MR $(\mathrm{P}=0.018)$, although in the case of LV end-diastolic volume it was not significant statistically $(\mathrm{P}=0.079)$. In contrast to LV global remodeling, there was no relationship between the severity of MR and tethering distance between the both PM tips and the contralateral anterior mitral annulus within the 3 groups (annular to anterior $\mathrm{PM}, \mathrm{P}=0.182$ and annular to posterior $\mathrm{PM}, \mathrm{P}=0.468$ ), suggesting no relationship between ischemic MR and local remodeling of $\mathrm{LV}$.

\section{Infarct Size}

Regarding CMR measurements, although there was a trend for increased total LV mass index and infarct transmurality in patients with higher degree of $\mathrm{MR}$, the association did not reach a statistical significance $(\mathrm{P}=0.409$ and $\mathrm{P}=0.064)$ (Table 3). However, there was a graded relationship between infarct mass index and the severity of MR $(\mathrm{P}=0.018)$. Also, patients with mild or moderate MR had larger infarct size

\begin{tabular}{|c|c|c|}
\hline & \multicolumn{2}{|c|}{$\begin{array}{l}\text { Model for the severity of MR } \\
\left(r^{2}=0.180, P=0.001\right)\end{array}$} \\
\hline & $\begin{array}{r}\text { Standardized } \\
\beta \text { coefficient }\end{array}$ & $P$ value \\
\hline LV ejection fraction (\%) & -0.264 & 0.022 \\
\hline Tenting area/BSA $\left(\mathrm{cm}^{2} / \mathrm{m}^{2}\right)$ & 0.255 & 0.027 \\
\hline
\end{tabular}

Abbreviations see in Tables 1,2.

than those without $\mathrm{MR}(\mathrm{P}=0.016)$.

\section{Determinants of the Severity of MR}

Multiple stepwise regression analysis revealed that significant independent predictors of the severity of MR were LV ejection fraction and tenting area $\left(\mathrm{r}^{2}=0.180, \mathrm{P}=0.001\right)$ (Table 4).

\section{Discussion}

The present study assessed the mechanism of MR during the acute phase of inferior wall MI and found that MR was associated with LV systolic dysfunction and tethering of mitral leaflet. Pathological tethering and concomitant regurgitation occur when an imbalance is present between closing and tethering forces. From the results of the current study, it can be speculated that decreased closing force in the presence of leaflet tethering would be the most important mechanism of MR in the acute phase of inferior wall MI.

Previously, a number of groups proved PM displacement tethered the mitral leaflets into the LV and restricted their ability to coapt effectively at the level of mitral annulus. It has been known that MR occurs in higher incidence for patients with inferior MI compared with those with anterior MI in spite of less LV remodeling, because localized inferior basal LV remodeling in patients with inferior MI can potentially cause greater geometric changes in the mitral valve apparatus with displacement of posterior PM, despite lesser global LV remodeling and dysfunction than that seen in patients with anterior MI.5-7 However, these series that underscored the localized inferior LV remodeling and PM displacement as the mechanism of ischemic MR included only patients in the chronic phase of MI. Therefore, we compared geometric changes and LV function between patients with or without MR after acute inferior MI within 1 week. From our data, it can be suggested that reduced closing force as a consequence of LV systolic dysfunction in the presence of leaflet tethering would play a more pivotal role in the development of MR in the acute phase of inferior MI, whereas increased tethering forces through a combination of annular dilation and geometric remodeling of the LV, especially PM displacement would be a more important contributor in the chronic phase (Figure 3). Our results are also consistent with previous study that LV dysfunction without 

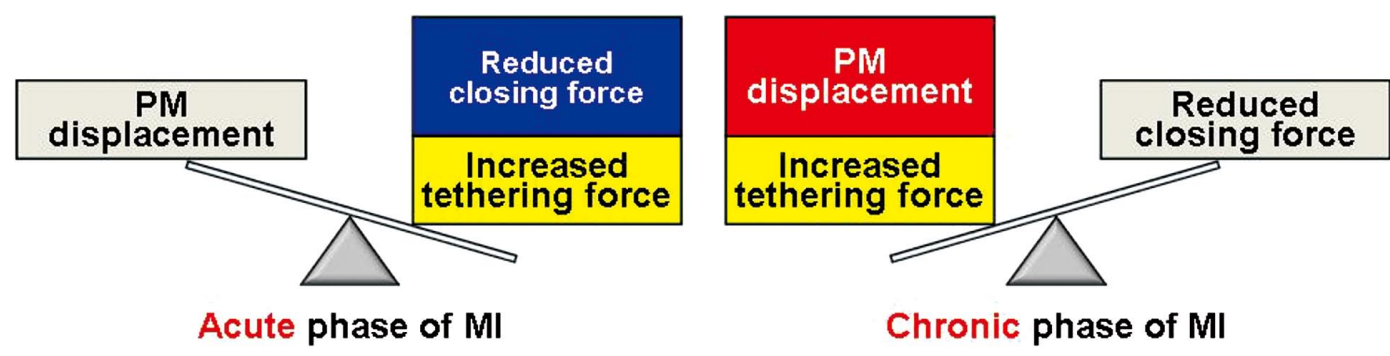

Figure 3. Reduced closing force as a consequence of left ventricular (LV) systolic dysfunction in the presence of leaflet tethering would play a more pivotal role in the development of mitral regurgitation in the acute phase of myocardial infarction (MI), whereas increased tethering force through a combination of annular dilation and geometric remodeling of the LV, especially papillary muscle displacement would be more important contributor in the chronic phase. Presence of tethering as an increased tethering force should be obligatory in both the acute and the chronic phase of MI. PM, papillary muscle.

dilatation fails to produce significant MR. ${ }^{18}$ Although LV systolic dysfunction was the most important factor as a determinant of the severity of MR, increased tenting area was also significant in multivariable regression analysis. Therefore, the presence of tethering as an increased tethering force should be obligatory in both the acute and the chronic phase of MI (Figure 3). Actually, global dilation of LV causes PM displacement and tenting to some extent. However, in our study LV dilation was not prominent to induce PM displacement because it is in the acute phase. Therefore, we believe that the combination of global dilation of LV (even though it is not sufficient to increase PM displacement), dilation of annular area and reduced closing force and so on can also increase tenting area from our results.

Although we excluded the patients who had any regional wall motion abnormality in the anterior wall to exclude any possible effect of ischemia of non-infarct related artery, such as left anterior descending artery, lack of the compensatory hypercontraction of the non-infarcted segments might be related to decreased LV global contractility. When we analyzed the data including the patients who had regional wall motion abnormality in the anterior wall, patients with mild and moderate MR had significantly increased RWSI in the anterior wall than those without MR (1.55 or 1.51 vs. 1.05 , $\mathrm{P}=0.001)$.

Based on these findings in our study, increasing closing force can be considered in the treatment of MR, especially in the acute phase of MI. Inotropic agents, such as dobutamine infusion, might reduce $\mathrm{MR}$ by raising $\mathrm{LV}$ pressure and increasing closing force. ${ }^{24,25}$ Diuretics or nitrates also can increase closing force by increasing transmitral pressure with decreasing LA pressure. ${ }^{26}$

Infarct size has been considered an established marker of LV remodeling. ${ }^{27,28}$ Therefore, we hypothesized that there would be a positive association between the severity of MR and infarct size if LV remodeling is an important mechanism of MR. Neskovic et al reported that peak creatine kinase value was significant independent predictors of MR and patients who developed MR had larger infarcts. ${ }^{29}$ To our knowledge, this would be the first study to show that infarct size measured by CMR has a positive relationship with MR during the acute phase of inferior wall MI. Not only systolic dysfunction but also LV remodeling in the patients with MR can be attributable to this larger infarct size measured by CMR.

$\mathrm{MR}$ in the acute phase of MI conveys adverse prognosis similarly in the chronic phase by doubling mortality after MI and the development of heart failure., ${ }^{2,30}$ Until now, it is certain that ischemic MR is an independent predictor of outcome, but the mechanism linking MR and the outcome is not well understood. Probably, the development of heart failure secondary to the development of ischemic MR is one of the main links between both entities. Thus, the development of ischemic MR leads to the development of heart failure and cardiac death. ${ }^{20,31}$ From the results of our study, it can be thought that the presence of ischemic MR in patients with inferior MI might be a reflector of poor LV function and remodeled LV, which is a substrate for adverse outcome after MI.

\section{Study Limitations}

There are several limitations of our study. First, the severity of MR was not assessed in a quantitative manner because we retrospectively reviewed the patients and quantitative assessment of the severity of MR was not routinely performed in patients with mild to moderate MR rather than severe, the data about MR severity in quantitative manner were not available in all patients. Second, estimation of geometric change in the mitral apparatus was done by using a single 2dimensional echocardiogram, and therefore we could not evaluate 3-dimensional PM displacement in multiple directions, as with 3-dimensional echocardiography. ${ }^{6,18,19}$ Third, our echocardiographic findings were observed after MI and do not exclude that some MR was present beforehand in some patients, a limitation shared by all studies on this topic. Fourth, we retrospectively reviewed the patients who performed CMR in the patients of MI and this would result in selection bias. Fifth, we did not quantify area at risk, which might be important for transient LV systolic dysfunction due to stunned myocardium as CMR performed in our patients had a protocol only to assess the presence and the extent of delayed enhancement. Sixth, we could not measure $+\mathrm{dP} / \mathrm{dt}$, a better parameter for LV contractility, as an index of LV contractility. Seventh, a small number of patients were studied.

\section{Conclusions}

In the acute phase of inferior wall MI, MR was associated with LV systolic dysfunction with tethering. Therefore, it can be suggested that reduced closing force as a consequence of LV systolic dysfunction in the presence of leaflet tethering would play a more pivotal role in the development of 
MR in the acute phase of inferior MI, whereas increased tethering forces through a combination of annular dilation and geometric remodeling of the LV would be more important contributor in the chronic phase.

\section{Acknowledgments}

We would like to thank Yutaka Otsuji, MD, for valuable comments on our manuscript.

\section{Disclosures}

Conflict of Interest: None declared. Sources of Funding: This study was supported by grants from the Korea Health 21 R\&D Project, Ministry of Health and Welfare, Republic of Korea (0412-CR02-0704-0001).

\section{References}

1. Bursi F, Enriquez-Sarano M, Jacobsen SJ, Roger VL. Mitral regurgitation after myocardial infarction: A review. Am J Med 2006; 119: $103-112$.

2. Lamas GA, Mitchell GF, Flaker GC, Smith SC Jr, Gersh BJ, Basta $\mathrm{L}$, et al. Clinical significance of mitral regurgitation after acute myocardial infarction: Survival and Ventricular Enlargement Investigators. Circulation 1997; 96: 827-833.

3. Grigioni F, Enriquez-Sarano M, Zehr KJ, Bailey KR, Tajik AJ. Ischemic mitral regurgitation: Long-term outcome and prognostic implications with quantitative Doppler assessment. Circulation 2001; 103: $1759-1764$.

4. Feinberg MS, Schwammenthal E, Shlizerman L, Porter A, Hod H, Friemark D, et al. Prognostic significance of mild mitral regurgitation by color Doppler echocardiography in acute myocardial infarction. Am J Cardiol 2000; 86: 903-907.

5. Kumanohoso T, Otsuji Y, Yoshifuku S, Matsukida K, Koriyama C, Kisanuki A, et al. Mechanism of higher incidence of ischemic mitral regurgitation in patients with inferior myocardial infarction: Quantitative analysis of left ventricular and mitral valve geometry in 103 patients with prior myocardial infarction. J Thorac Cardiovasc Surg 2003; 125: 135-143.

6. Watanabe N, Ogasawara Y, Yamaura Y, Yamamoto K, Wada N, Kawamoto T, et al. Geometric differences of the mitral valve tenting between anterior and inferior myocardial infarction with significant ischemic mitral regurgitation: Quantitation by novel software system with transthoracic real-time three-dimensional echocardiography. J Am Soc Echocardiogr 2006; 19: 71-75.

7. Arikawa K, Otsuji Y, Zhang BY, Uemura T, Hamasaki S, Biro S, et al. Left ventricular remodeling is less while left atrial remodeling is greater in inferior compared to anterior myocardial infarction: Importance of ischemic mitral regurgitation. $J$ Echocardiogr 2004; 2: $43-48$.

8. Pennell DJ, Sechtem UP, Higgins CB, Manning WJ, Pohost GM, Rademakers FE, et al. Clinical indications for cardiovascular magnetic resonance (CMR): Consensus Panel report. Eur Heart J 2004; 25: $1940-1965$.

9. Ishida M, Kato S, Sakuma H. Cardiac MRI in ischemic heart disease. Circ J 2009; 73: 1577-1588.

10. Schiller NB, Shah PM, Crawford M, DeMaria A, Devereux R, Feigenbaum H, et al. Recommendations for quantitation of the left ventricle by two-dimensional echocardiography: American Society of Echocardiography Committee on Standards, Subcommittee on Quantitation of Two-Dimensional Echocardiograms. J Am Soc Echocardiogr 1989; 2: 358-367.

11. Cerqueira MD, Weissman NJ, Dilsizian V, Jacobs AK, Kaul S, Laskey WK, et al. Standardized myocardial segmentation and nomenclature for tomographic imaging of the heart: A statement for healthcare professionals from the Cardiac Imaging Committee of the Council on Clinical Cardiology of the American Heart Association. Circulation 2002; 105: 539-542.

12. D'Cruz IA, Shroff SG, Janicki JS, Jain A, Reddy HK, Lakier JB. Differences in the shape of the normal, cardiomyopathic, and volume overloaded human left ventricle. J Am Soc Echocardiogr
1989; 2: 408-414.

13. Ren JF, Kotler MN, DePace NL, Mintz GS, Kimbiris D, Kalman P, et al. Two-dimensional echocardiographic determination of left atrial emptying volume: A noninvasive index in quantifying the degree of nonrheumatic mitral regurgitation. J Am Coll Cardiol 1983; 2: 729-736.

14. Garcia MJ, Thomas JD, Klein AL. New Doppler echocardiographic applications for the study of diastolic function. J Am Coll Cardiol 1998; 32: 865-875.

15. Ormiston JA, Shah PM, Tei C, Wong M. Size and motion of the mitral valve annulus in man. I: A two-dimensional echocardiographic method and findings in normal subjects. Circulation 1981; 64: $113-120$.

16. Vijayaraghavan G, Boltwood CM, Tei C, Wong M, Shah PM. Simplified echocardiographic measurement of the mitral anulus. Am Heart J 1986; 112: 985-991.

17. Godley RW, Wann LS, Rogers EW, Feigenbaum H, Weyman AE. Incomplete mitral leaflet closure in patients with papillary muscle dysfunction. Circulation 1981; 63: 565-571.

18. Otsuji Y, Handschumacher MD, Schwammenthal E, Jiang L, Song JK, Guerrero JL, et al. Insights from three-dimensional echocardiography into the mechanism of functional mitral regurgitation: Direct in vivo demonstration of altered leaflet tethering geometry. Circulation 1997; 96: 1999-2008.

19. Liel-Cohen N, Guerrero JL, Otsuji Y, Handschumacher MD, Rudski LG, Hunziker PR, et al. Design of a new surgical approach for ventricular remodeling to relieve ischemic mitral regurgitation: Insights from 3-dimensional echocardiography. Circulation 2000; 101: $2756-2763$.

20. Otsuji Y, Handschumacher MD, Liel-Cohen N, Tanabe H, Jiang L, Schwammenthal E, et al. Mechanism of ischemic mitral regurgitation with segmental left ventricular dysfunction: Three-dimensional echocardiographic studies in models of acute and chronic progressive regurgitation. J Am Coll Cardiol 2001; 37: 641-648.

21. Helmcke F, Nanda NC, Hsiung MC, Soto B, Adey CK, Goyal RG, et al. Color Doppler assessment of mitral regurgitation with orthogonal planes. Circulation 1987; 75: 175-183.

22. Freed LA, Levy D, Levine RA, Larson MG, Evans JC, Fuller DL, et al. Prevalence and clinical outcome of mitral-valve prolapse. $N$ Engl J Med 1999; 341: 1-7.

23. Orn S, Manhenke C, Anand IS, Squire I, Nagel E, Edvardsen T, et al. Effect of left ventricular scar size, location, and transmurality on left ventricular remodeling with healed myocardial infarction. Am J Cardiol 2007; 99: 1109-1114.

24. Keren G, Katz S, Strom J, Sonnenblick EH, LeJemtel TH. Dynamic mitral regurgitation: An important determinant of the hemodynamic response to load alterations and inotropic therapy in severe heart failure. Circulation 1989; 80: 306-313.

25. Heinle SK, Tice FD, Kisslo J. Effect of dobutamine stress echocardiography on mitral regurgitation. J Am Coll Cardiol 1995; 25: $122-127$.

26. Levine RA, Schwammenthal E. Ischemic mitral regurgitation on the threshold of a solution: From paradoxes to unifying concepts. Circulation 2005; 112: 745-758.

27. Tarantini G, Razzolini R, Cacciavillani L, Bilato C, Sarais C, Corbetti $\mathrm{F}$, et al. Influence of transmurality, infarct size, and severe microvascular obstruction on left ventricular remodeling and function after primary coronary angioplasty. Am J Cardiol 2006; 98: 1033-1040.

28. Yasuda S, Shimokawa H. Acute myocardial infarction: The enduring challenge for cardiac protection and survival. Circ J 2009; 73: 2000-2008.

29. Neskovic AN, Marinkovic J, Bojic M, Popovic AD. Early predictors of mitral regurgitation after acute myocardial infarction. Am J Cardiol 1999; 84: 329-332, A328.

30. Lehmann KG, Francis CK, Dodge HT. Mitral regurgitation in early myocardial infarction. Incidence, clinical detection, and prognostic implications: TIMI Study Group. Ann Intern Med 1992; 117: 1017.

31. Perez de Isla L, Zamorano J, Quezada M, Almeria C, Rodrigo JL, Serra V, et al. Functional mitral regurgitation after a first non-STsegment elevation acute coronary syndrome: Contribution to congestive heart failure. Eur Heart J 2007; 28: 2866-2872. 